\title{
Conf-940723-35
}

UCRL-JC-117673

PREPRINT

\section{Remote Measurement of Ground Temperature and Emissivity}

John R. Henderson

This paper was prepared for submittal to the Society of Photo-Optical Instrumentation Engineers San Diego, CA

July 25-28, 1994

\section{June 1994}

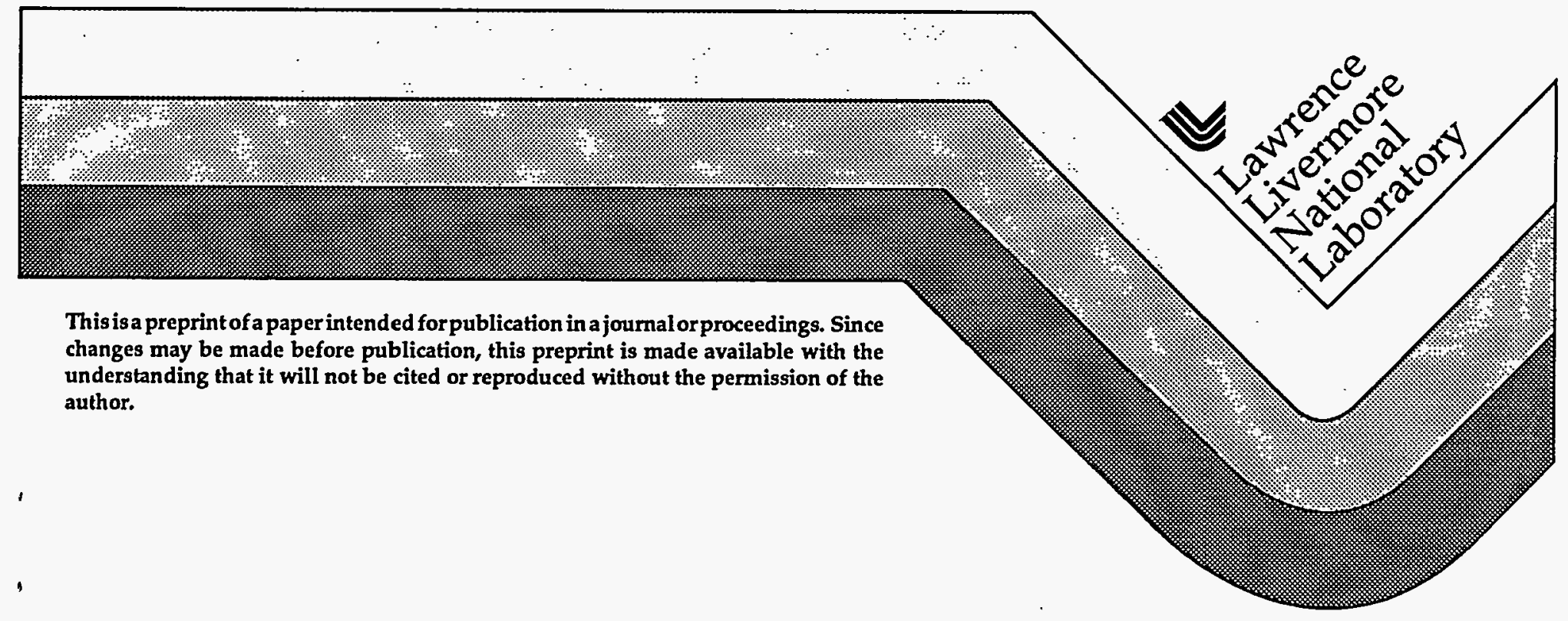




\section{DISCLAIMER}

This document was prepared as an account of work sponsored by an agency of the United States Government. Neither the United States Government nor the University of California nor any of their employees, makes any warranty, express or implied, or assumes any legal liability or responsibility for the accuracy, completeness, or usefulness of any information, apparatus, product, or process disclosed, or represents that its use would not infringe privately owned rights. Reference herein to any specific commercial products, process, or service by trade name, trademark, manufacturer, or otherwise, does not necessarily constitute orimply its endorsement, recommendation, or favoring by the United States Government or the University of California. The views and opinions of authors expressed herein do not necessarily state or reflect those of the United States Government or the University of California, and shall not be used for advertising or product endorsement purposes. 


\section{DISCLAIMER}

Portions of this document may be illegible in electronic image products. Images are produced from the best available original document. 


\title{
Remote Measurement of Ground Temperature and Emissivity
}

\author{
John R. Henderson \\ Physics and Space Technology, MS L-043 \\ Lawrence Livermore National Laboratory \\ Livermore, California 94550
}

\begin{abstract}
TAISIR , Temperature and Imaging System InfraRed, is a nominally satellite based platform for remote sensing of the earth. One of its design features is to acquire atmospheric data simultaneous with ground data, resulting in minimal dependence on external atmospheric models for data correction. Extensive modeling of the rms error of determining a ground temperature and emissivity for a gray body has been performed as a function of integration time, spectroscopic resolution of the system, ground emissivity, atmospheric variables, and atmospheric data accuracy. We find that increased resolution improves measurement accuracy by emphasizing those regions where the atmospheric transmission is highest and atmospheric emission/absorption lowest. We find rms temperature errors $\leq 1 \mathrm{~K}$ and rms emissivity errors $<0.01$ are obtainable for reasonable seeing and with sufficient information about the atmosphere. A new method is developed for modeling the dependence of the bandaveraged transmission and emission. Monte Carlo simulations of satellite data taken using a multi-angle technique are used to derive signal-to-noise requirements. The applicability of those results to. the TAISIR system requirements are discussed.
\end{abstract}

Keywords: thermometry, infrared, imaging, atmospheric modeling, multi-angle, atmospheric transmission, band-averaged transmission

\section{INTRODUCTION}

The goal of the Temperature and Imaging System InfraRed (TAISIR) project is to measure ground temperatures in a robust manner, that is, to retrieve ground temperatures even under conditions of poor seeing and with little or no model dependence. The basic TAISIR concept is to use a low resolution imager with a co-aligned high resolution spectrometer. A third component of the TAISIR research program is the development of novel data acquisition techniques. The imager data will be used to provide imagery and perform thermometry on ground sources using spectral bands to obtain the IR emission spectrum from the source. The information required to fully exploit the imager data is the atmospheric emission and transmission, and some constraints on the source emissivity, diffuse reflectivity, or specular reflectivity. Historically, radiosonde or weather satellite information has been used to obtain the atmospheric properties through modeling codes. The accuracy of ground temperature retrieval has been limited to a few degrees by: the accuracy with which the modeling codes

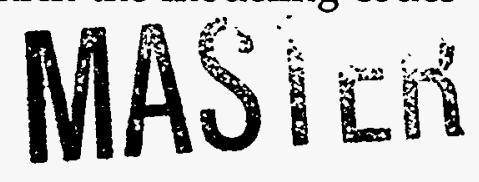


correctly describe the atmosphere; use of non-ideal weather data due to lack of concurrent and/or co-spatial information; and uncertainties in the ground source emissivity due to the lack of direct measurement.

The use of multiple look angles (angular sweep technique) provides data with a systematic variation between signal and depth of atmospheric column that can be used to extract most of the required atmospheric information. The remaining information can be obtained from modeling, where the required quantities have minimal sensitivity to the model inputs, or from measurements of line intensities from the spectrometer. This paper focuses on developing the techniques to exploit angular sweep data, and their application to imager data.

The early data from the feasibility studies for TAISIR are used to motivate subsequent work and derive some useful signal-to-noise $(S / N)$ requirements. Multiple angle techniques are discussed and several methods of treating the band-averaged properties of the atmosphere are developed. One of these methods is used to derive the relationships between system S/N and the accuracy to which the atmospheric emission and transmission can be determined. Finally, the important results are summarized and directions for future research are outlined.

\section{PREVIOUS WORK}

A Monte Carlo simulation of the basic TAISIR design was performed to ensure that there were no fundamental physics limitations to the acquisition of high $\mathrm{S} / \mathrm{N}$ data. A single layer model of the atmosphere was used in conjunction with high resolution $\left(0.2 \mathrm{~cm}^{-1}\right)$ measurements of atmospheric emission to generate lower resolution atmospheric emission and transmission. The transmission was calculated using $\tau=1-\varepsilon_{\mathrm{a}}$, where $\tau$ and $\varepsilon_{\mathrm{a}}$ are the transmission and emissivity for each waveband. The emissivity was calculated from

$$
\varepsilon_{\mathrm{a}}=\mathrm{L}_{\mathrm{a}} / \mathrm{B}_{v}\left(\mathrm{~T}_{\mathrm{a}}\right),
$$

where $L_{a}$ is the atmospheric emission and $B_{v}\left(T_{a}\right)$ is the black body emission at the effective atmospheric temperature $T_{a}$. The source emission, $L_{s}$, was calculated from a source temperature and gray body emissivity, $\varepsilon_{\mathrm{S}}$ and propagated through the atmosphere to obtain the measured signal at the detector. Integration time, solid angle, and detector quantum efficiency were included to correctly account for counting statistics. In addition, adjustable uncertainties were included for $\mathrm{T}_{\mathrm{a}}$ and the measured atmospheric emission. A Gaussian random number generator was used to generate noise in the source emission, the atmospheric emission and the atmospheric transmission. The signal at the detector, ignoring reflected atmospheric radiation, is then

$$
\mathrm{L}_{\mathrm{det}}=\tau * \mathrm{~L}_{\mathrm{s}}+\mathrm{L}_{\mathrm{a}} \text {, }
$$

which inverts to give

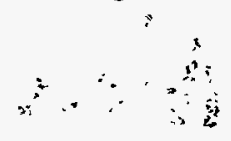




$$
\mathrm{L}_{\mathrm{s}}=\left(\mathrm{L}_{\mathrm{det}}-\mathrm{L}_{\mathrm{a}}\right) / \tau
$$

The quantity $L_{s}$ was obtained from equation 3 using the noise-free values for $\tau$ and $L_{a}$. Standard error propagation was used to obtain uncertainty in $\mathrm{L}_{s}$ in each waveband. Finally, a non-linear least squares fit was done to $L_{s}(\lambda)=\varepsilon_{s} * B_{v}\left(T_{s}, \lambda\right)$ to obtain both $\varepsilon_{s}$ and $T_{s} . B_{v}\left(T_{s}, \lambda\right)$ is the black body emission at wavelength $\lambda$ for an object of temperature $T_{s}$. Table 1 shows the results of interest here.

Table 1. Uncertainties in the fitted source temperature and emissivity as a function of the rms uncertainty in the atmospheric temperature for a single layer model atmosphere.

\begin{tabular}{|c|c|c|}
\hline $\mathrm{rms} \mathrm{Ta}$ & sigma Ts & $\operatorname{sigma} \varepsilon$ \\
\hline 0.0 & 0.018 & $<0.001$ \\
\hline 0.1 & 0.041 & $<0.001$ \\
\hline 0.3 & 0.038 & 0.001 \\
\hline 1.0 & 0.215 & 0.003 \\
\hline 3.0 & 0.456 & 0.007 \\
\hline 10.0 & 2.670 & 0.040 \\
\hline
\end{tabular}

For uncertainties in $\mathrm{T}_{\mathrm{a}}$ greater than $0.2 \mathrm{~K}$, the fitting uncertainty is dominated by the uncertainty in $\mathrm{T}_{\mathrm{a}}$. Requiring the ability to measure ground temperatures to $1 \mathrm{~K}$ limits the uncertainty in $\mathrm{T}_{\mathrm{a}}$ to about $5 \mathrm{~K}$. The variation of $\mathrm{T}_{\mathrm{a}}$ in the simulation only affects the transmission $\tau$. It can be shown that, for a single layer atmosphere with known emission,

$$
\frac{\partial \tau}{\partial \mathrm{T}_{\mathrm{a}}} \approx(1-\tau) *\left(\frac{1.439 \mathrm{e} 4 \mathrm{~K}-\mu \mathrm{m}}{\lambda * \mathrm{~T}_{\mathrm{a}}}\right) * \frac{1}{\mathrm{~T}_{\mathrm{a}}}
$$

where $\lambda$ is in $\mu \mathrm{m}$. The measured data were taken under high visibility conditions with corresponding peak transmissions of $\sim 90 \%$. This puts a limit of \pm 0.010 on $\tau$. For more typical peak transmissions of $50 \%$ to $80 \%$, the limit on the uncertainty in $\tau$ becomes 0.05 to 0.02 .

The uncertainty in the emission from the atmosphere also affects the temperature retrieval. A. change of $1 \mathrm{~K}$ in a $300 \mathrm{~K}$ black body produces a change in flux of $1.6 \%$ at $10 \mu \hat{\mathrm{m}}$. Assuming a transmission of about one-half, the maximum allowed uncertainty in the atmospheric emission is $0.8 \%$ of that due to a $300 \mathrm{~K}$ black body. We thus have the requirement of determining both the atmospheric emission and transmission to better than $1 \%$. 
In this simulation, it was assumed that the atmospheric information was available from some outside source. This is the typical case when using atmospheric models such as MODTRAN to invert satellite data. It is easy to see how the presence of undetected sub-visual cirrus, for example, can introduce errors of several degrees ${ }^{1}$ by altering the transmission and sky emission by several percent. It is essential to have a system that takes all needed atmospheric information simultaneous with the imaging data.

\section{MULTIPLE ANGLE TECHNIQUES}

\subsection{Background and basic phenomena}

Techniques using multiple (specifically two) viewing angles to enhance remote measurements were proposed ${ }^{2}$ at least as early as 1967 . The technique exploits the variation of the transmission and the emission of the atmosphere with the variation of the depth of the atmospheric column. As the viewing angle deviates from the nadir, the column depth increases, the atmospheric emission increases, and the flux seen at a remote sensor decreases for a ground source warmer than the atmosphere. A wide range of accuracies are reported using the technique: Chedin $e t \mathrm{al}^{3}$ obtain rms deviations of $1 \mathrm{~K}$ to $2 \mathrm{~K}$ simulating satellite observations of sea surfaces, Macleod 4 obtained 0.3K for an airborne platform, and Byrnes and Schott ${ }^{5}$ measured rms errors of $1.0 \mathrm{~K}$ to $6.6 \mathrm{~K}$ at altitudes of 1000 feet to 6000 feet, respectively. Simulations by $\operatorname{Cogan}^{6}$ support the magnitude and linear scaling with altitude observed. Section 3.3 discusses one shortcoming of these techniques.

The physics of multi-angle viewing through the atmosphere can be illustrated by considering several cases. For a totally transmitting atmosphere all one sees is the ground emission and there is no variation with angle. For a totally opaque atmosphere, all one sees is the upper layer of the atmosphere, and again there is no variation with angle. At either extreme, there will be little variation with angle. Consider a waveband with a transmission of $70 \%$. Doubling the path length will drop the transmission to 50\%. (Treat the new path as two layers of $70 \%$ transmission each). Since the transmission is lower, the emissivity of the atmosphere is higher and one will see a smaller IR flux if the ground is warmer than the atmosphere.

Naively, one would expect that the best source information would be obtained for transmissions of $50 \%$ to $70 \%$. In section 4 , we show that the lowest absolute error in the transmission is obtained for the lowest transmissions. This is because there is the greatest variation with atmospheric depth for small transmission. Let $\tau=1-\delta$. The variation with relative column depth, $l$, can be expanded as

$$
\tau^{l}=(1-\delta)^{l} \approx\left(1-l^{*} \delta\right) .
$$


To lowest order, the greatest dependence of the transmission on column depth occurs for the largest $\delta$, hence the smallest transmission. Since the greatest source information is obtained for the largest transmissions, we expect the optimum transmission to be around 0.5 .

\subsection{Naive model of band-averaged transmission}

The standard development of multi-angle techniques uses a band-averaged transmission, $\tau$. Taking $\theta$ to be the angle from nadir, the transmission as a function of angle is given by

$$
\tau=\tau_{0}{ }^{1 / \cos \theta}=\tau_{0}{ }^{\sec \theta}=\tau_{0}^{l},
$$

where $\tau_{0}$ is the transmission at nadir and $l$ is the relative depth of the atmosphere. For a nonreflective material (the atmosphere) the emissivity and transmissivity are related by

$$
\tau=1-\varepsilon
$$

The atmospheric emission, $\mathrm{L}_{a}$ is given by

$$
\mathrm{L}_{\mathrm{a}}=\varepsilon_{\mathrm{a}} * \mathrm{~B}_{v}\left(\mathrm{~T}_{\mathrm{a}}, \lambda_{1}, \lambda_{2}\right),
$$

where $B_{v}\left(T_{a}, \lambda_{1}, \lambda_{2}\right)$ is the black body emission for a source at temperature $T_{a}$ over the waveband from $\lambda_{1}$ to $\lambda_{2}$. Hereafter, the explicit wavelength dependence will be suppressed and we will use the shorthand $B_{a}=B_{v}\left(T_{a}, \lambda_{1}, \lambda_{2}\right)$, and $B_{s}=B_{v}\left(T_{s}, \lambda_{1}, \lambda_{2}\right)$ for the atmosphere and source black body emission.

The ground is assumed to have an emissivity $\varepsilon_{\mathbf{S}}$, specular reflectivity $R s$, and diffuse reflectivity $R d$, which are related by

$$
i=\varepsilon_{s}+R_{s}+R_{d} .
$$

The ground emission is then,

$$
L_{s}=\varepsilon_{S} * B s+R_{d} * \varepsilon_{A} * B_{a}+R_{S} * \varepsilon_{a} * B_{a} \text { ， }
$$

where $\varepsilon_{\mathrm{A}}$ is the angular average of the sky emissivity, and it has been assumed that the downwelled sky emission is equal to the upwelled sky emission. $\varepsilon_{\mathbf{A}}$ is a function of $\tau_{0}$ and the scene geometry. Combining equations $2,6,7,8$ and 10, we have the expression for the detected flux, $\mathrm{L}_{\mathrm{d}}$, as a function of relative atmospheric depth 


$$
\begin{aligned}
\mathrm{L}_{\mathrm{d}}(l) & =\left(1-\tau_{0}^{l}\right)^{*} \mathrm{~B}_{\mathrm{a}}+\left(\tau_{0}^{l}\right) *\left[\varepsilon_{\mathrm{S}} * \mathrm{~B}_{\mathrm{S}}+\mathrm{R}_{\mathrm{d}} * \varepsilon_{\mathrm{A}} * \mathrm{~B}_{\mathrm{a}}+\mathrm{R}_{\mathrm{s}} *\left(1-\tau_{0}^{l}\right) * \mathrm{~B}_{\mathrm{a}}\right], \\
& =\mathrm{B}_{\mathrm{a}}+\left[\varepsilon_{\mathrm{s}} * \mathrm{~B}_{\mathrm{S}}+\mathrm{R}_{\mathrm{d}} * \varepsilon_{\mathrm{A}} * \mathrm{~B}_{\mathrm{a}}+\mathrm{R}_{\mathrm{s}} * \mathrm{~B}_{\mathrm{a}}-\mathrm{B}_{\mathrm{a}}\right] * \tau_{0}^{l}-\mathrm{R}_{\mathrm{s}} * \mathrm{~B}_{\mathrm{a}} * \tau_{0}{ }^{2 l}, \\
& =\mathrm{A}+\mathrm{B} * \tau_{0}^{l}+\mathrm{C} * \tau_{0}^{2 l},
\end{aligned}
$$

where

$$
\begin{aligned}
& A=B_{a}, \\
& B=\varepsilon_{s} * B_{s}+R_{d} * \varepsilon_{A} * B_{a}+R_{s} * B_{a}-B_{a}, \\
& C=-R_{s} * B_{a} .
\end{aligned}
$$

Since there are four fit parameters, $A, B, C$, and $\tau_{0}$, and one constraining equation (Eq. 9), and seven parameters of interest, $B_{\mathrm{S}}, \varepsilon_{\mathrm{S}}, R_{\mathrm{d}}, R_{\mathrm{S}}, B_{\mathrm{S}}, \varepsilon_{\mathrm{S}}, \tau_{0}$, the problem is underconstrained for any single waveband. (Recall that $\varepsilon_{A}$ is a function of $\tau_{0}$.) Since $T_{S}$ is independent of wavelength, $\mathrm{T}_{\mathrm{a}}$ nearly so (depending on the average optical depth in the band), and there are generally some constraints on the ground properties, such as slow variation of $\varepsilon_{\mathrm{S}}$ with wavelength, the problem is constrained for multiple wavebands.

\subsection{First order model of band-averaged transmission}

The shortcoming of the naive model is that equation 6 is not a realistic description of how the band-averaged transmission varies with relative atmospheric depth. Figure 1 compares the transmission from a MODTRAN run for a $60^{\circ}$ satellite view ( $67^{\circ}$ ground angle) scaled by the relative depth (2.554) to the transmission at nadir at $2 \mathrm{~cm}^{-1}$ resolution. The ratio is near one for regions where there are few lines, but the scaling is seriously flawed where there are any strong lines. The problem is that there are opaque regions in the band where the transmission is zero, and equation 6 may apply only to the transmissive regions. This suggests considering the opaque and transmissive regions separately. Let $\mathrm{f}_{\mathbf{1}}$ be the fraction of the waveband that is transmissive and $\tau_{1}$ be the average transmission there. Then the depth-dependent transmission, $\tau(l)$, is given by

$$
\tau(l)=f_{1} *\left(\tau_{1}^{l}\right),
$$

and the atmospheric emission is given by

$$
L_{a}=f_{1} *\left(1-\tau_{1} l\right) * B_{a}+\left(1-f_{1}\right) * B_{0},
$$




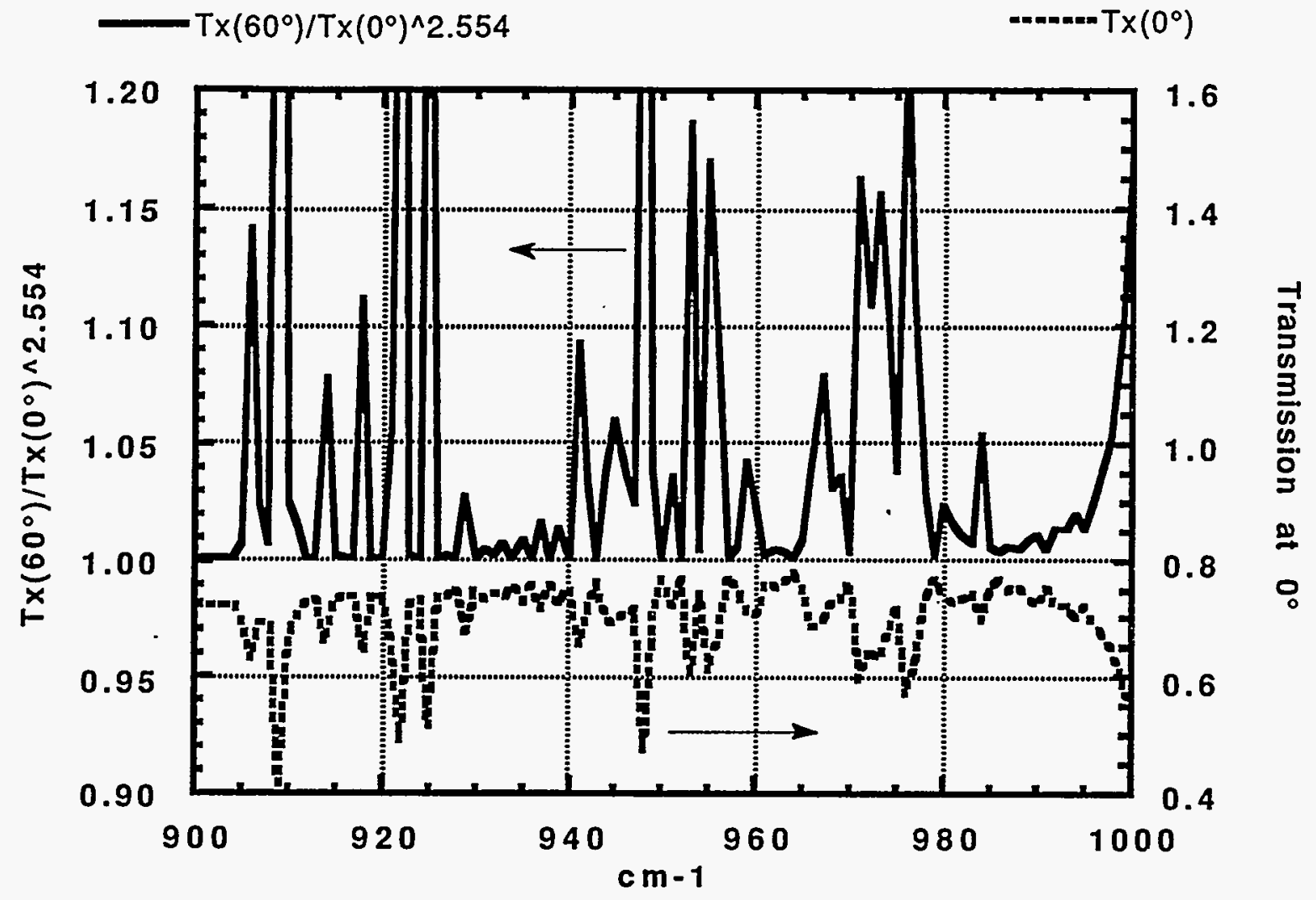

Figure 1. MODTRAN results demonstrating the failure of the naive model to properly account for the variation of transmission with depth in the atmosphere. These results are at $1 \mathrm{~cm}^{-1}$. Broader bands include more line structure and show larger deviations from unity. The lower curve is the transmission at nadir.

where $B_{0}$ is the emission for a black body at the temperature, $T_{0}$, of the opaque layer. (By definition, the opaque layer has an emissivity on one.) Comparing to the previous section, we find that

$$
\mathrm{f}_{1}^{*} \tau_{1}=\tau_{0}
$$

For two atmospheric depths, we have

$$
\mathrm{f}_{1}^{*} \tau_{1}^{2}=\tau_{0}^{2} / \mathrm{f}_{1}>\tau_{0}^{2}
$$

since $f 1<1$. For strong lines $f_{1}$ is relatively small, so the transmission will be larger than calculated in the naive model. This is seen in Figure 1. 
The flux seen at a detector can be derived as in the previous section. The result is

$$
\mathrm{L}_{\mathrm{d}}(l)=\mathrm{A}+\mathrm{B} * \tau_{0} l+\mathrm{C} * \tau_{0}^{2 l},
$$

where

$$
\begin{aligned}
& A=f_{1} * B_{a}+\left(1-f_{1}\right) * B_{0}, \\
& B=\varepsilon_{s} * B_{s}+R_{d} * \varepsilon_{A} * B_{a}+R_{s} *\left(1-f_{1}\right) * B_{o}+R_{s} * f_{1} * B_{a}-B_{a}, \\
& C=-f_{1}{ }^{2 * R_{s} * B_{a} .}
\end{aligned}
$$

Since we have the same number of constraints as before, but have added a wavelength dependent parameter $f_{1}$ and a nominally wavelength-independent parameter $T_{0}$ (giving $B_{0}(\lambda)$ ), the problem is now underdetermined. Since $f_{1}$ depends primarily on the fraction of strong lines in the waveband, and secondarily on their strength, one would expect $f_{1}$ to have only a weak dependence on the precise amount of the absorbing agent. Figure 2 shows the results of several MODTRAN runs comparing $f_{1}$ for a wide variety of atmospheric conditions. The variations on absorption lines are only a few percent for variations of a factor of three in the water content. The variations are negligible between lines. The spectral region shown is in the middle of the transmission window. The variations are about twice as large at the edges of the absorption window. Determining $f_{1}$ is the only model dependence in this algorithm, and that dependence is quite weak. Alternatively, the spectrometer could be used to measure the strength of calibration lines, and measure $\mathrm{f}_{1}$ directly.

\subsection{Second order model of band-averaged transmission}

The first order model barely meets the requirements outlined in section 2 . Calculations of $f_{1}$ from MODTRAN runs show that $\mathrm{f1}$ varies by about $10 \%$ on lines at $1 \mathrm{~cm}^{-1}$ resolution and by about $1 \%$ everywhere at $10 \mathrm{~cm}^{-1}$ resolution. Physically, the limitation is that as the atmospheric depth is increased, some lines become opaque, effectively reducing $\mathrm{f}_{1}$.

The shortcoming is easily corrected by allowing for a distribution of transmissions in the band. In the first order model, the probability distribution of transmissivities is a step function. In the second order model, it is essentially a smoothed step function. Assuming a normal distribution around the transition, we have

$$
\tau(l)=\tau_{2} l * \int_{0}^{1}\left\{0.5-0.5 * \operatorname{sgn}(x) *\left[1-\exp \left(\frac{-2 x^{2}}{\pi}\right)\right]^{1 / 2}\right\}^{l} d f
$$

where $x=\left[\left(f-f_{2}\right) / \omega_{2}\right]$, and the formula is an approximation ${ }^{7}$ to the normal probability function 


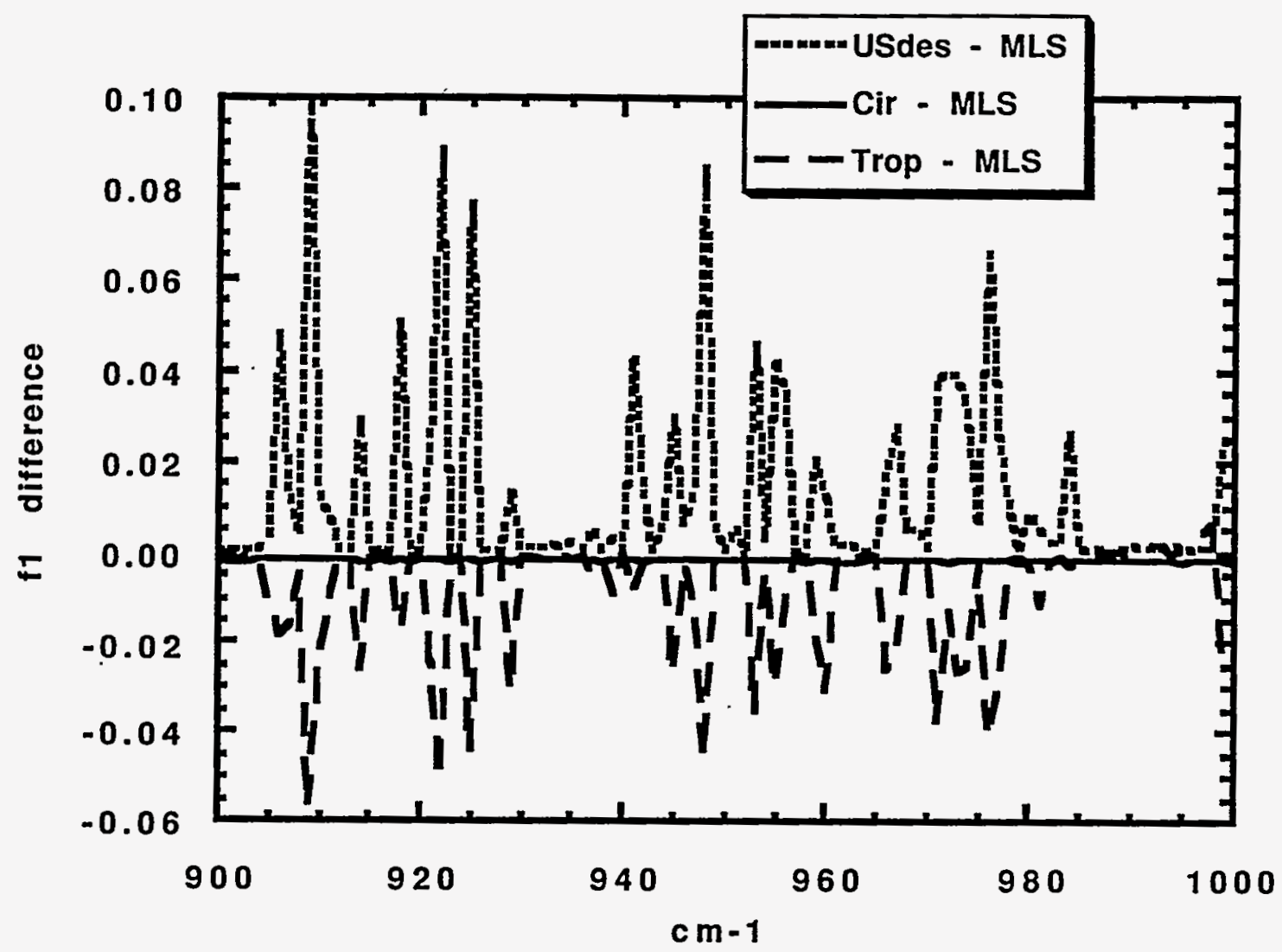

Figure 2. Variation in $f_{1}$ for a variety of atmospheric conditions. Values are derived from MODTRAN calculated transmissions. Mid-latitude summer conditions were taken as the reference. The water vapor content varies a factor of three over the plot.

that is good to $0.4 \%$. For $l=1$, this reduces to

$$
\tau(l=1)=\mathrm{f}_{2} * \tau_{2}=\tau_{0},
$$

which is identical to the relationship in the first order model. Comparison of equations 22 and 15 show that development of a second order model will be much more computationally intensive. Numerical evaluation of equation 22 shows that it does result in a lower effective 1 with increasing $l$, as needed to improve the first order model.

\section{4. "DATA" INVERSION: DEPENDENCE ON SIGNAL-TO-NOISE}

The simplest case to explore is that where the ground has an emissivity of one. Normalizing the fluxes to an effective sky temperature of $273 \mathrm{~K}$, equations 18 to 21 become 


$$
\mathrm{L}_{\mathrm{d}}(l)=\mathrm{A}+\mathrm{B} * \tau_{1} l,
$$

where

$$
\begin{aligned}
& A=1, \\
& B=f_{1} *\left[\left(B_{S} / B_{a}\right)-1\right] .
\end{aligned}
$$

Since $f_{1}$ and $\tau_{1}$ are somewhat correlated, the parameter space can be explored by varying $f_{1}$ and $\tau_{1}$ together, and varying $\left(B_{S} / B_{a}\right)$ independently. Typical values for $f 1$ are $0.6,0.95$, and 1.00 for regions at the edge of the transmission window, in the bulk of the window, and at peak transmission regions, respectively. Similarly, $\tau_{1}$ has typical values of $0.4,0.75$, and 0.9 . Ratios for $\left(\mathrm{B}_{\mathrm{s}} / \mathrm{B}_{\mathrm{a}}\right)$ of $1.10,1.61$, and 2.18 correspond to ground temperatures of $278 \mathrm{~K}, 300 \mathrm{~K}$, and $320 \mathrm{~K}$. Noise was added to the "signal" from equation 24 by using a Gaussian random number scaled by the inverse of the $\mathrm{S} / \mathrm{N}$. For a satellite fly-over the atmospheric depth varies from one at nadir to two at $60^{\circ}$ to three at $70.5^{\circ}$. For these studies, the upper limit on $l$ was varied from 2.0 to 3.0. The number of observations was fixed at 12. More observations will reduce the random uncertainties. The procedure used was to generate a series of "detected" fluxes for a range of depths that correspond to uniform satellite motion. This series of $L_{d}$ as a function of $l$ was inverted to obtain $A, B$, and $\tau_{1}$.

Inversion of equation 24 requires non-linear techniques, which are much slower than matrix inversion using linear techniques. Assuming that most of the important information would be acquired in the high transmission regions, $\tau_{1}$ was written as $(1-\delta)$ and equation 24 expanded to third order in $\delta$ and $l$. The approximation agrees with the exact form to at least $1 \%$ for all $l<2.0$ and for all $\delta<0.5$. A set of "data" were generated using equation 24 as described above. The data were inverted with the cubic approximation, and the fit parameters determined. Each data set was repeated 100 times to accumulate statistics on the variability of the fit parameters. Table 2 shows the minimum $S / N$ required to obtain an accuracy of at least \pm 0.01 in $A, B$, and $\tau_{1}$ for maximum atmosphere depths of $2.0,2.5$, and 3.0.

\section{DISCUSSION REGARDING TEMPERATURE RETRIEVAL}

The results of Table 2 indicate the need for high $\mathrm{S} / \mathrm{N}$ to obtain adequate information about the atmospheric emission and transmission to obtain ground temperatures to an accuracy of $1 \mathrm{~K}$ when the sweep angle technique is used alone. Since multiple wavebands will be used, the $\mathrm{S} / \mathrm{N}$ in any waveband does not have to be this high, as long as the noise is random and not systematic. The use of additional information will also relax the $S / N$ requirements compared to the results here. For example, when $T_{S}=T_{a}$ no variation with depth will be observed. Consequently, there is no leverage to retrieve the transmission, and a large $\mathrm{S} / \mathrm{N}$ requirement 
Table 2. Minimum $S / N$ required for desired accuracy in the fit parameters as a function of the maximum atmospheric depth, $l_{\max }$. The fimiting parameter is indicated.

\begin{tabular}{|c|c|c|c|}
\hline$l_{\max }=2.0$ & $\mathrm{Ts}=278 \mathrm{~K}$ & $\mathrm{Ts}=300 \mathrm{~K}$ & $\mathrm{Ts}=320 \mathrm{~K}$ \\
\hline$f_{1}, \tau_{1}=0.6,0.4$ & $1 \mathrm{e} 5\left(\tau_{1}\right)$ & $2 \mathrm{e} 4\left(\tau_{1}\right)$ & $\operatorname{le} 4\left(A, \tau_{1}\right)$ \\
\hline $\mathrm{f}_{1}, \tau_{1}=0.95,0.75$ & $3 \mathrm{e} 5\left(\tau_{1}\right)$ & $2 \mathrm{e} 5(\mathrm{~A}, \mathrm{~B})$ & $2 \mathrm{e} 5(\mathrm{~A}, \mathrm{~B})$ \\
\hline$f_{1}, \tau_{1}=1.0,0.9$ & $>1 e 6$ (all) & $>1 \mathrm{e} 6(\mathrm{~A}, \mathrm{~B})$ & $>1 \mathrm{e} 6(\mathrm{~A}, \mathrm{~B})$ \\
\hline$l_{\max }=2.5$ & $\mathrm{Ts}=278 \mathrm{~K}$ & $\mathrm{Ts}=300 \mathrm{~K}$ & $\mathrm{Ts}=320 \mathrm{~K}$ \\
\hline$f_{1}, \tau_{1}=0.6,0.4$ & $4 \mathrm{e} 4\left(\tau_{1}\right)$ & $8 \mathrm{e} 3\left(\tau_{1}\right)$ & $3 \mathrm{e} 3\left(\mathrm{~A}, \tau_{1}\right)$ \\
\hline$f_{1}, \tau_{1}=0.95,0.75$ & $1 \mathrm{e} 5\left(\tau_{1}\right)$ & $4 \mathrm{e} 4(\mathrm{~A}, \mathrm{~B})$ & $5 \mathrm{e} 4(\mathrm{~A}, \mathrm{~B})$ \\
\hline$f_{1}, \tau_{1}=1.0,0.9$ & $\sim 5 e 5$ (all) & $\sim 5 \mathrm{e} 5(\mathrm{~A}, \mathrm{~B})$ & $\sim 7 \mathrm{e} 5(\mathrm{~A}, \mathrm{~B})$ \\
\hline$l_{\max }=3.0$ & $\mathrm{Ts}=278 \mathrm{~K}$ & $\mathrm{Ts}=300 \mathrm{~K}$ & $\mathrm{Ts}=320 \mathrm{~K}$ \\
\hline$f_{1}, \tau_{1}=0.6,0.4$ & $2 \mathrm{e} 4\left(\tau_{1}\right)$ & $3 \mathrm{e} 3\left(\tau_{1}\right)$ & $1 \mathrm{e} 3\left(\mathrm{~A}, \tau_{1}\right)$ \\
\hline $\mathrm{f}_{1}, \tau_{1}=0.95,0.75$ & $4 \mathrm{e} 4\left(\tau_{1}\right)$ & $2 \mathrm{e} 4(\mathrm{~A}, \mathrm{~B})$ & $3 \mathrm{e} 4(\mathrm{~A}, \mathrm{~B})$ \\
\hline $\mathrm{f}_{1}, \tau_{1}=1.0,0.9$ & 2e5 (all) & $2 \mathrm{e} 5(\mathrm{~A}, \mathrm{~B})$ & $-3 e 5(A, B)$ \\
\hline
\end{tabular}

will be indicated. However, the fact that no variation with depth is observed implies that there is a minimal temperature difference. Poor retrieval of the transmission is irrelevant (and impossible!) in that case, but still generates the highest $\mathrm{S} / \mathrm{N}$ requirement. As shown in Eq. 5, the best atmospheric information (as indicated by smallest required $S / N$ ) is obtained for the lowest transmissions. This ability to acquire high quality atmospheric information under nonoptimal conditions is the core of the robustness of the data acquisition for TAISIR. Since the best source information is obtained for the highest transmissions, the tradeoff between the two needs to be explored for band selection. A full simulation of the problem and the data inversion is required to address these system issues.

The noise requirements are relaxed considerably as the maximum column depth is increased. Additional work needs to be done to include the bending in the atmosphere and effects from the earth's curvature.

\section{CONCLUSIONS AND FUTURE WORK}

An improved model for band-averaged transmission and emission has been developed and successfully applied to some of the design requirements for TAISIR. The sweep angle technique has been shown to provide robust retrieval of atmospheric information. It has been 
shown that the noise requirements imposed by a simple nadir-looking model will overestimate the required $\mathrm{S} / \mathrm{N}$ for a sweep angle data collection technique.

Development of a system simulation including a variety of wavebands and a full data inversion is necessary to accurately determine the system requirements. The system performance and simulation results will be verified by flights on a high-altitude balloon.

\section{ACKNOWLEDGMENTS}

I wish to thank Charlie Bennett for many very enlightening discussions throughout this work. Many members of the TAISIR team have been helpful in identifying the key physics issues to address, particularly Charles Hailey, Mike Carter, and Dave Fields. Deanne Proctor has been of great help running MODTRAN. This work was performed under the auspices of the U.S. Department of Energy by Lawrence Livermore National Laboratory under contract No. W7405-Eng-48.

\section{REFERENCES}

1. W. M. Cornette and J. G. Shanks, "Impact of Cirrus Clouds on Remote Sensing of Surface Temperatures," in Passive Infrared Remote Sensing of Clouds and the Atmosphere, SPIE Proc. 1934, pp. 252-263, April 1993.

2. P. M. Saunders, "Aerial Measurements of Sea Surface Temperature in the Infrared," J. Geophys. Res., 72, pp. 4109 - 4117, 1967.

3. A. Chedin, N. A. Scott, and A. Berroir, "A Single Channel Double-Viewing Angle Method for Sea Surface Temperature Determination from Coincident METEOSAT and TIROS-N Radiometric Measurements," J. Appl Meterol., 15, p. 173, 1976.

4. I. D. Macleod, "An Airborne Thermal Remote Sensing Technique," M.S. Thesis, Rochester Institute of Technology, Rochester, NY, 1983.

5. A. E. Byrnes and J. R. Schott, "Correction of thermal imagery for atmospheric effects using aircraft measurement and atmospheric modeling techniques," Appl. Opt., 25, pp. 2563 - 2570, August 1986.

6. J. L. Cogan, "Passive remote sensing of slant path transmittance," Appl. Opt., 27, pp 32803289, August 1988.

7. M. Abramowitz and I. A. Stegun, Handbook of Mathematical Functions, equation 26.2.24, Dover Publications, New York, 1965. 